\title{
Regional Myocardial Blood Flow in Awake Dogs
}

\author{
Frederick R. Cobb, Robert J. Bache, and Joseph C. Greenfield, Jr. \\ From the Veterans Administration Hospital, Durham, North Carolina 27705 \\ and the Department of Medicine, Division of Cardiology, Duke University \\ Medcal Center, Durham, North Carolina 27710
}

A в S T R A C T The objectives of this study were to test the hypothesis in awake dogs that during control conditions endocardial vessels are maximally dilated and to determine whether variables introduced by general anesthesia and thoracotomy modify distribution of myocardial blood flow or impair capacity for augmentation of flow in response to a coronary vasodilator stimulus. Myocardial blood flow was measured in relatively small, 2-3 g, left ventricular epicardial and endocardial samples by using $7-10-\mu \mathrm{m}$ radioisotope-labeled microspheres during control conditions and during infusion of adenosine in dosages which produced maximum increases in coronary blood flow. Measurements were made initially in awake resting animals and were repeated after pentobarbital anesthesia, thoracotomy, and pericardiotomy.

Blood flow (mean \pm SEM) in the epicardium and endocardium, respectively, was $0.75 \pm 0.06$ and $0.83 \pm 0.06$ during control conditions and $4.98 \pm 0.28$ and $4.49 \pm 0.27 \mathrm{~cm}^{3}$, $\mathrm{min} / \mathrm{g}$ during adenosine. These data demonstrate considerable capacity for vasodilation in both myocardial layers and thus refute the hypothesis that endocardial vessels are maximally dilated during control conditions. During control conditions blood flow within epicardial and endocardial layers was essentially homogeneous around the circumference of the left ventricle. In contrast to previous studies in anesthetized animals, however, transmural gradients were present in most regions, i.e., endocardium: epicardium ratio (endo/epi) 1.06-1.16. During adenosine, circumferential epicardial flows were homogeneous; however, circumferential endocardial flows were inhomogeneous and increased less than epicardial flows, endo/epi 0.81-0.99.

Anesthesia, thoracotomy, and pericardiotomy increased epicardial and endocardial flow, mean values $1.08 \pm 0.10$ and $1.11 \pm 0.08 \mathrm{~cm}^{3} / \mathrm{min} / \mathrm{g}$, respectively. Transmural

Drs. Cobb and Bache are Clinical Investigators, Veterans Administration Hospital, Durham, N. C. Dr. Greenfield is recipient of Research Career Development Award 1-K3HL-28,112 from the U. S. Public Health Service.

Received for publication 8 October 1973 and in revised form 21 December 1973. gradients remained in only papillary muscle regions. Adenosine increased epicardial flow comparably before and after anesthesia. Although adenosine increased endocardial flow three- to fourfold after anesthesia, the increase was considerably less than epicardial flow, i.e., endo/epi 0.63-0.78.

\section{INTRODUCTION}

Measurements of transmural myocardial blood flow and the capacity to augment endocardial blood flow have yielded conflicting results (1-10). Winbury (1), using polarographic techniques for measuring intramyocardial oxygen tension in anesthetized dogs, reported lower $\mathrm{Po}_{2}$ within the subendocardium than in the subepicardium. From these data he concluded that the subendocardium was chronically underperfused and that endocardial arterial resistance was low and fixed. In support of this view, Kirk and Honig (2) and Brandi, Fam, and McGregor (3), using depot clearance techniques, reported that blood flow in deep myocardial layers was $20-25 \%$ less than flow in superficial layers. However, other investigators using either diffusable indicators (4-7) or 7-10- $\mu \mathrm{m}$ radioisotope-labeled microspheres $(8,9)$, have reported that blood flow in the endocardium and epicardium was comparable. Moir (10) hypothesized that endocardial perfusion is maintained by a transmural gradient of coronary vascular resistance during diastole which compensates for underperfusion of the endocardium during systole. He reasoned that compensatory vasodilation during diastole may decrease the capacity for further vasodilation in the endocardium and that such a mechanism may be a factor which accounts for the greater vulnerability of the subendocardial region to ischemic injury.

The purpose of this study was to measure circumferential and transmural blood flow in small left ventricular samples, 2-3 g, using 7-10- $\mathrm{m}$ microspheres during basal and maximal blood flow rates and thus to test the hypothesis that the endocardial vessels are maximally dilated during control conditions with no capacity for fur- 
ther vasodilation. Accordingly, blood flow measurements were made in awake trained dogs during quiet, resting control conditions and during infusion of adenosine in dosages which produced maximum increases in coronary blood flow (11).

Many of the previous studies which have measured transmural myocardial blood flow have been performed in acute surgical preparations. Since the coronary vascular system is sensitive to external interventions, it is possible that variables introduced by anesthesia and acute surgical stress and trauma may have influenced the normal reactivity of the coronary vasculature and biased the estimates of myocardial blood flow. To test the hypothesis that variables introduced by utilizing acute surgical preparations significantly alter control flow and the capacity to increase flow, each animal was subjected to pentobarbital anesthesia, mechanical ventilation, thoracotomy, and pericardiotomy and measurements were repeated before and during adenosine infusion.

\section{METHODS}

13 mongrel dogs weighing $25-33 \mathrm{~kg}$ were anesthetized with sodium pentobarbital $30 \mathrm{mg} / \mathrm{kg}$ and ventilated with a respirator. $^{1}$ A left thoracotomy was performed via the fourth intercostal space. A polyvinyl chloride catheter, $3 \mathrm{~mm} \mathrm{OD}$, was inserted into the left atrial cavity via the atrial appendage and secured with a purse-string suture. A polyvinyl chloride catheter, $3.5 \mathrm{~mm} \mathrm{OD}$, was inserted into the root of the aorta via the left internal thoracic artery. Both catheters were filled with heparin and tunneled to the base of the neck and placed in a subcutaneous pouch.

Studies were performed 7-10 days after the surgical procedure at which time each animal was free of signs of infection. The mean hematocrit at the time of study was $46 \pm 4.7$, range $39-50 \%$. During the recovery period each animal became accustomed to the laboratory environment and was trained to lie quietly on a table. On the day of the study the left atrial and aortic pressure catheters were exteriorized from the subcutaneous pouch using 2\% lidocaine infiltration anesthesia. The arterial catheter was attached to a pressure transducer ${ }^{2}$ and a lead II of a standard electrocardiogram also was recorded. Hemodynamic data were recorded on a direct-writing oscillographic system. Studies were performed with the animals lying on their right side and loosely restrained. After all recording instruments were connected, a $45-60-\mathrm{min}$ period was allowed for each animal to adjust to the laboratory conditions. During this period, the laboratory was dimly illuminated and all noise and activity were minimized. Under these conditions the animals were inactive and appeared to doze.

Measurements of myocardial blood flow were made by serial injections of carbonized microspheres $7-10-\mu \mathrm{m}$ in diameter labeled with gamma-emitting nuclides ${ }^{51} \mathrm{Cr}$, ${ }^{141} \mathrm{Ce}$, ${ }^{85} \mathrm{Sr}$, and ${ }^{46} \mathrm{Sc} .{ }^{4}$ The microspheres were obtained as 1 $\mathrm{mCi}$ of each nuclide in $10 \mathrm{~cm}^{3}$ of $10 \%$ dextran and $0.05 \%$ polysorbate 80 . The stock solution was diluted in $10 \%$ dex-

${ }^{1}$ Model 607, Harvard Apparatus Co., Inc., Millis, Mass. ${ }^{2}$ P23Db Transducer, Statham Laboratories, Inc., HatoRey, Puerto Rico.

${ }^{3}$ Model 7700, Hewlett-Packard Co., Palo Alto, Calif.

‘3M Co., St. Paul, Minn. tran so that each $\mathrm{cm}^{3}$, the volume injected, contained approximately two million microspheres. This dosage was selected so that during control conditions each gram of myocardium would receive a minimum of approximately 500 microspheres, i.e., assuming a coronary blood flow of $5 \%$ of the cardiac output and heart weight of $150-200 \mathrm{~g}$. Serial injection of this quantity of microsphere resulted in no change in heart rate or blood pressure in the dogs included in the study. Blood pressure was measured before and immediately after collection of the reference sample. Changes in arterial pressure during the reference sample collection which were not accompanied by changes in heart rate could not be excluded. However, in awake animals, even slight changes in blood pressure are usually accompanied by measureable reflex changes in heart rate. Heart rate increased and blood pressure decreased transiently after the injection in one dog not included in the study. In five additional awake animals, not used in the present study, with electromagnetic flow probes implanted on the aorta and left circumflex coronary artery, four sequential injections of two million microspheres did not affect coronary or aortic blood flow or the reactivity of the coronary vasculature as measured by the response to a brief ischemic stimulus. Before each injection the microspheres were thoroughly mixed by alternate agitation for at least $15 \mathrm{~min}$ in an ultrasonic bath ${ }^{\mathbf{5}}$ and vortex agitator. Complete dispersion was verified by microscopic examination of a drop of the mixture. $1 \mathrm{~cm}^{3}$ of the microsphere mixture was then injected into the left atrium over a 15-s interval via the previously implanted catheter and flushed in with $5-10 \mathrm{~cm}^{3}$ of warm isotonic saline. During each injection a reference sample was collected from the aortic arch via the previously implanted catheter. Collection of the reference sample was begun simultaneously with the beginning of microsphere injection and continued for $90 \mathrm{~s}$. Reference blood samples were collected in previously weighed counting tubes. Reference blood samples were collected at rates of $20 \mathrm{~cm}^{3} / \mathrm{min}$, by using a withdrawal pump. ${ }^{6}$ The volume collected during a 60-s interval was accurately measured by weighing the counting tubes. A blood specific gravity of 1.06 was used for converting each weighed reference flow sample to volume. Over the range of hematocrits measured in the present study, the blood specific gravity should vary less than $1 \%$. The hematocrit did not change during the course of each study. There were no counts above background in the reference samples collected after $60 \mathrm{~s}$ in any measurements included in the present study.

Measurements of myocardial blood flow were made during quiet resting conditions, before, and during maximum coronary vasodilation produced by an i.v. infusion of adenosine, $4.0 \mu \mathrm{mol} / \mathrm{kg}$ of body weight $/ \mathrm{min}$ via the right antecubital vein. The adenosine was dissolved in warmed normal saline, $30 \mu \mathrm{mol} / \mathrm{cm}^{3}$. The total infused volume was approximately $20 \mathrm{~cm}^{3}$. Previous studies in this laboratory in which left circumflex coronary artery blood flow was measured in awake animals by using electromagnetic flow probes demonstrated that this dosage of adenosine resulted in maximum increases of coronary blood flow since $(a)$ no further increase in flow was observed during infusion of larger dosages and $(b)$ no further increase in flow was observed in response to brief periods of total occlusion, i.e., the reactive hyperemic response was completely obliterated. The adenosine infusion was begun $3 \mathrm{~min}$ before

"Model DA0950, 3M Co.

'Model 1210, Harvard Apparatus Co., Inc. 


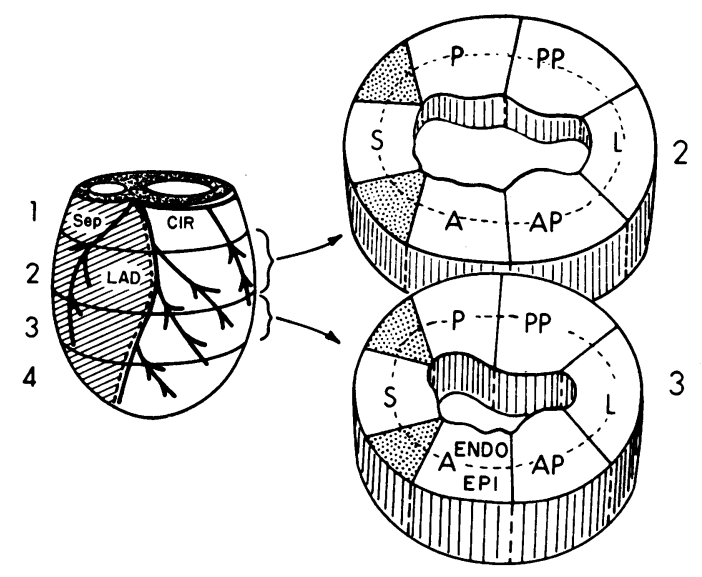

FIGURE 1 Illustrates the technique for sectioning the left ventricle. The atrial tissue and right ventricle were removed, as indicated by the stippled and lined area in the left hand figure. The left ventricle was then sectioned into four transverse sections and the two central sections, 2 and 3 , were subdivided into anterior $(A)$, septum $(S)$, posterior $(P)$, posterior papillary $(P P)$, lateral $(L)$, and anterior papillary $(A P)$ regions. Each region was further divided into equal epicardial (epi) and endocardial (endo) regions.

injection of the microspheres in order to achieve a steady state during the hemodynamic study.

Each animal was then anesthetized with pentobarbital, 30 $\mathrm{mg} / \mathrm{kg}$, and ventilated with a respirator. ${ }^{1}$ A left thoracotomy was performed via the intercostal space below the previous incision and the pericardium was opened. Injection of microspheres was again performed before and during a similar infusion of adenosine. Microspheres were injected during control conditions in 13 dogs both awake and after anesthesia and during adenosine infusion in 11 awake and 9 anesthetized dogs. The nuclide used for each injection was selected randomly.

In three anesthetized dogs a catheter ${ }^{7}$ was introduced into the left external jugular vein and positioned in the coronary sinus under fluoroscopic control. A $10-\mathrm{cm}^{3}$ coronary sinus blood sample was obtained during each microsphere injection. Radioactivity found in the coronary sinus blood sample during control and maximum flow rates represented less than $1.0 \%$ of the corresponding radioactivity found in the myocardium.

At the completion of each study the animal was killed with a lethal dose of pentobarbital. The heart was removed, weighed, and placed in $10 \%$ formalin for a 3-day period to facilitate sectioning. The heart weight was $181 \pm 32 \mathrm{~g}$ (mean $\pm \mathrm{SD})$. The atrial tissue, right ventricle, pericardial fat, aorta, and large surface blood vessels were then dissected from the left ventricle and discarded. The mean weight of the left ventricle was $113 \pm 19 \mathrm{~g}$. The ventricle was sectioned into four transverse sections of approximately equal thickness (Fig. 1). The two central sections which constituted $51 \pm 6 \%$ of the left ventricular weight were then divided into six regional areas, i.e., anterior, septum, posterior, posterior papillary muscle, lateral, and anterior papillary muscle. Each area was then divided into equal outer

${ }^{7}$ No. 7 French Sones, U. S. Catheter and Instrument Co., Glens Falls, N. Y. (epicardial) and inner (endocardial) layers (Fig. 1), weighed, and placed in vials for counting. For the remainder of this presentation, these layers will be referred to as "epicardium" and "endocardium." The weights of individual samples ranged from 0.98 to $4.48 \mathrm{~g}$, with most samples weighing 2-3 g. Epicardial sample weights were slightly greater than endocardial sample weights, i.e, the mean weight of samples from each epicardial and endocardial region, respectively, ranged from 1.95-3.38 $\mathrm{g}$ and 1.48-2.83 g. The myocardial and reference blood samples were counted in a gamma spectrometer ${ }^{8}$ at optimum window settings selected to correspond to the peak energies of each nuclide. The counts per minute recorded in each window from each myocardial and reference sample were corrected for overlapping counts of the accompanying isotopes by using a digital computer. ${ }^{\ominus}$ Blood flow to each area of myocardium in $\mathrm{cm}^{3} / \mathrm{min}$ was calculated by using the formula :

$$
F_{m}=F_{r} \cdot C_{m} / C_{r}
$$

where $F_{m}=$ myocardial flow $\mathrm{cm}^{3} / \mathrm{min}, F_{r}=$ reference flow $\mathrm{cm}^{3} / \mathrm{min}, C_{m}=$ counts $/ \mathrm{min}$ in myocardium, and $C_{r}=$ counts $/$ min in reference flow. Myocardial flow, $\mathrm{cm}^{3} / \mathrm{min}$, was divided by the sample weight and expressed as $\mathrm{cm}^{3} / \mathrm{min} / \mathrm{g}$.

A rough estimate of coronary vascular resistance was calculated by dividing mean arterial pressure by mean flow/100 $\mathrm{g}$ of myocardium.

The variability of blood flow in small adjacent areas of the myocardium was evaluated by calculating the flow differences between each pair of adjacent endocardial and between each pair of adjacent epicardial samples. The endocardial and epicardial differences between each pair of adjacent samples were averaged separately and the $95 \%$ confidence limits computed, assuming a normal distribution. The range of differences was also included to further describe the data variability.

Multivariate analysis using a Hotelling $\mathrm{T}^{2}$ test was used to determine if blood flow in comparable epicardial and endocardial segments of rings 2 and 3 were different (12). If differences were found, paired $t$ tests were used to determine which segments in ring 2 were different from the comparable segments in ring 3 . The $P$ values were adjusted by the Bonferonni inequality (13) which corrects for performing multiple tests on correlated data, i.e, each $P$ value was multiplied by the number of paired $t$ tests performed on each set of data and a $P$ value of 0.05 was required for statistical significance. Comparable regions in rings 2 and 3 which were not different were averaged. The Hotelling $\mathrm{T}^{2}$ test was used to determine if blood flow in different regions within the endocardium or epicardium was different. If differences were found, paired $t$ tests were used to determine which regional flow was different trom flow in the anterior region. The $P$ values were adjusted by the Bonferonni inequality.

The distribution of flow between the endocardium and epicardium was compared by testing if the ratio of endo/ epi flow was different from 1.0. The Hotelling $\mathrm{T}^{2}$ test on the logarithms of the flow ratio was used to test this hypothesis. Multiple paired $t$ tests modified by the Bonferroni inequality were used to determine which individual segments were different.

\footnotetext{
${ }^{8}$ Model 167776, Beckman Instruments, Inc., Fullerton, Calif.

${ }^{\ominus}$ Model 1130, International Business Machines Corp., Armonk, N. Y.
} 
The effect of each intervention on blood flow was tested by using both the Hotelling $\mathrm{T}^{2}$ test and multiple adjusted paired $t$ tests. Blood flow values before and after anesthesia and surgery were compared in 13 dogs. Measurements before and during adenosine were compared in 11 of the 13 dogs before and nine dogs after anesthesia and surgery. The adenosine response during resting conditions was compared to the adenosine response after anesthesia and surgery in nine dogs (Fig. 2).

\section{RESULTS}

In 13 animals during quiet resting conditions, values (mean \pm SEM), were as follows: heart rate $71 \pm 3$ beats/ min, blood pressure $100 \pm 3 \mathrm{~mm} \mathrm{Hg}$, epicardial blood flow $0.75 \pm 0.06 \mathrm{~cm}^{3} / \mathrm{min} / \mathrm{g}$, and endocardial blood flow $0.83 \pm 0.06 \mathrm{~cm}^{3} / \mathrm{min} / \mathrm{g}$. In order to assess the variability of flow measurements between adjacent myocardial samples, the mean difference in blood flow between the 173 pairs of adjacent epicardial samples was computed and found to be $-1.0 \%$ with $95 \%$ confidence limits of $\pm 20 \%$. The range of differences was -34 to $+28 \%$. In the 173 pairs of adjacent endocardial samples, the mean difference was $+2.0 \%, 95 \%$ confidence limits $\pm 20 \%$, and a range of -29 to $+27 \%$.

Blood flow during the control state in comparable epicardial and endocardial regions of rings 2 and 3 were not different statistically, with the exception of the septal endocardium where blood flow in ring 2 was significantly greater than, in ring 3 . Values for comparable regions in rings 2 and 3, except the septal endocardial samples,

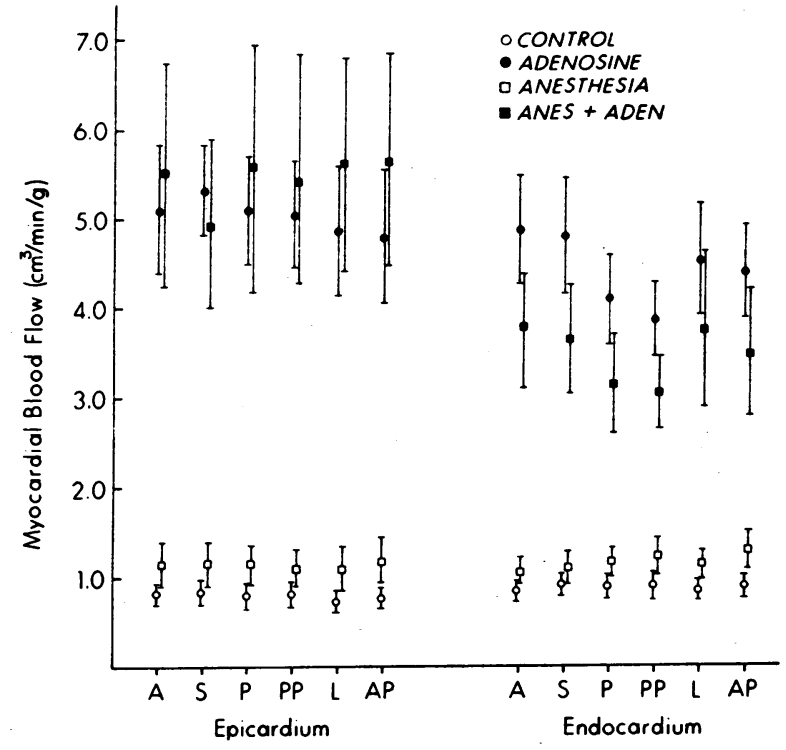

Figure 2 Illustrates the mean myocardial blood flow values, $\mathrm{cm}^{3} / \mathrm{min} / \mathrm{g}( \pm 2 \mathrm{SEM})$, from the various epicardial and endocardial regions during basal flow rates and during maximum flow rates produced by i.v. adenosine $4.0 \mu \mathrm{mol} /$ $\mathrm{kg} / \mathrm{min}$ in awake resting dogs. These measurements were repeated after anesthesia, thoracotomy, and pericardiotomy.

were averaged in each dog and were subjected to multivariate analysis to test for possible differences in flow between various regions around the circumference of the

TABLE I

Regional Myocardial Blood Flow

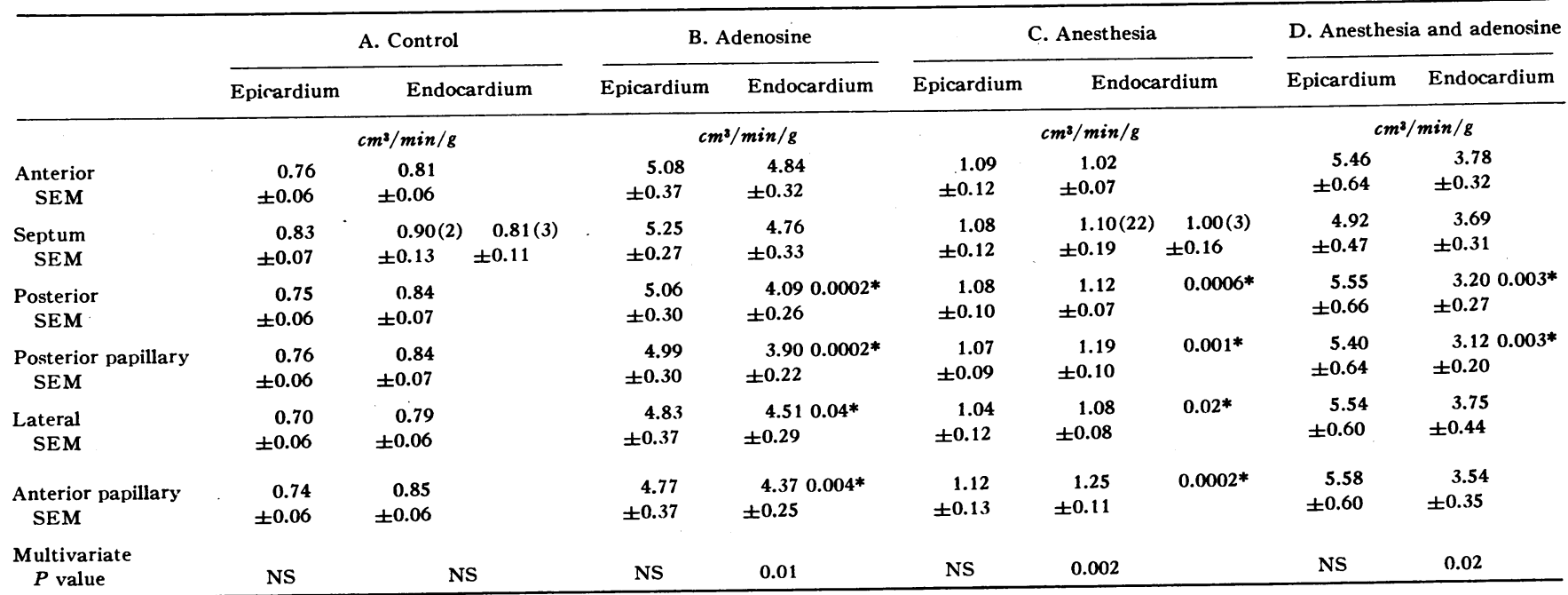

Tabulation of mean blood values, $\mathrm{cm}^{3} / \mathrm{min} / \mathrm{g}( \pm \mathrm{SEM})$, in the anterior, septal, posterior, posterior papillary, lateral, and anterior papillary regions. Measurements were made in quiet resting animals (A) before, (B) during an i.v. infusion of adenosine, $4.0 \mu \mathrm{mol} / \mathrm{kg}$ of body weight/min. Measurements were repeated after anesthesia,

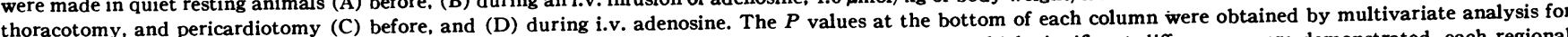
difference between measurement in each myocardial layer, significance $=P<0.05$. In the layers in which significant differences were demonstrated, each regional value was compared to blood flow in the anterior sample, significant differences are indicated by the asterisk and the $P$ values listed. The adjacent values from rings 2 and 3 in the septal endocardial region were different statistically during interventions $A$ and $C$ and thus were not averaged and were not included in the multivariate analysis. 
TABLE II

Endocardium/Epicardium Blood Flow Ratios

\begin{tabular}{ccccc}
\hline & A. Control & B. Adenosine & C. Anesthesia & $\begin{array}{c}\text { D. Anesthesia } \\
\text { and adenosine }\end{array}$ \\
\hline Anterior & $1.06 \pm 0.03$ & $0.99 \pm 0.08$ & $0.95 \pm 0.03$ & $0.75 \pm 0.04$ \\
$P$ & $\mathrm{NS}$ & $\mathrm{NS}$ & $\mathrm{NS}$ & 0.04 \\
Septum & $1.06 \pm 0.03$ & $0.91 \pm 0.04$ & $0.99 \pm 0.03$ & $0.78 \pm 0.03$ \\
$P$ & $\mathrm{NS}$ & $\mathrm{NS}$ & $\mathrm{NS}$ & 0.001 \\
Posterior & $1.12 \pm 0.04$ & $0.83 \pm 0.05$ & $1.06 \pm 0.04$ & $0.63 \pm 0.04$ \\
$P$ & 0.04 & 0.04 & $\mathrm{NS}$ & 0.0006 \\
Posterior papillary & $1.11 \pm 0.03$ & $0.81 \pm 0.05$ & $1.12 \pm 0.02$ & $0.65 \pm 0.06$ \\
$P$ & 0.02 & 0.02 & 0.04 & 0.004 \\
Lateral & $1.16 \pm 0.03$ & $0.99 \pm 0.09$ & $1.07 \pm 0.04$ & $0.73 \pm 0.05$ \\
$P$ & 0.006 & $\mathrm{NS}$ & $\mathrm{NS}$ & 0.01 \\
Anterior papillary & $1.16 \pm 0.03$ & $0.95 \pm 0.08$ & $1.13 \pm 0.04$ & $0.68 \pm 0.05$ \\
$P$ & 0.0006 & $\mathrm{NS}$ & 0.02 & 0.004 \\
\hline
\end{tabular}

Tabulation of endocardium/epicardium blood flow ratios ( \pm SEM) in the anterior, septum, posterior, posterior papillary, lateral, and anterior papillary regions. Measurements were made in quiet resting animals (A) before and (B) during an intravenous infusion of adenosine. Measurements were repeated after anesthesia, thoracotomy, and pericardiotomy (C) before, and (D) during intravenous adenosine. $P$ values test whether the ratio is significantly different from 1.0.

ventricle (see column A; Table I). No significant differences were found between adjacent and nonadjacent regional blood flow in either the epicardium or endocardium. Blood flow to the septal endocardium in rings 2 and 3 was greater than and equal to anterior endocardial flow, respectively. Mean endocardial (endo) flow was greater than mean epicardial (epi) flow in each region, i.e., endo/epi ratios varying from 1.06 to 1.16 , with ratios statistically greater than 1.0 in the posterior, posterior papillary, lateral, and anterior papillary regions (column A, Table II). Thus, in the awake dog during quiet resting conditions, variability of individual blood flow measurements between adjacent regions in the endocardium and epicardium was small, adjacent and nonadjacent regional blood flow measurements within each layer were not different, and significant transmural blood flow gradients which favored the endocardium were present in most regions.

In 11 awake dogs i.v. infusion of adenosine produced a significant increase in heart rate and a decrease in arterial pressure, mean values were $111 \pm 5$ beats $/ \mathrm{min}(P<$ $0.01)$ and $86 \pm 3 \mathrm{~mm} \mathrm{Hg}(P<0.01)$, and a marked increase in blood flow to both the epicardium and endocardium, mean values were $4.98 \pm 0.28$ and $4.49 \pm 0.27$ $\mathrm{cm}^{3} / \mathrm{min} / \mathrm{g}$, respectively. Mean coronary vascular resistance during adenosine infusion was $13 \%$ and $16 \%$ of the control epicardial and endocardial values. Mean difference in blood flow between the 143 pairs of adjacent epicardial samples was $0.4 \%$ with $95 \%$ confidence limits of $\pm 28 \%$. The range was -55 to $+47 \%$. In the 143 pairs of adjacent endocardial samples, the mean difference was $4.0 \%, 95 \%$ confidence limits $\pm 28 \%$, and range -27 to $+43 \%$.

Blood flow values during adenosine infusion in comparable epicardial and endocardial regions of rings 2 and 3 , including the septal regions, were not different statistically. Thus, these were averaged in each dog and then subjected to multivariate analysis to test for differences in regional blood flow (column $\mathrm{B}$, Table $\mathrm{I}$ ). No significant differences were found between regional blood flow in the epicardium; however, significant differences were present in the endocardium, i.e., posterior, posterior papillary, lateral, and anterior papillary endocardial flows were significantly less than anterior endocardial flow (see column B, Table I). Adenosine produced changes in the transmural distribution of blood flow. During maximum flow rates produced by adenosine the control transmural gradient was decreased in the anterior wall and septum regions, obliterated in the lateral and anterior papillary regions, and was reversed in the posterior and posterior papillary regions (column B, Table II). Although both the epicardium and endocardium demonstrated a marked capacity to augment blood flow and decrease coronary vascular resistance during adenosine infusion, the capacity was significantly less in the endocardium, resulting in an obliteration or reversal of the normal transmural blood flow gradient and significant differences in regional blood flow within the endocardium. 
After each of the 13 control dogs was subjected to anesthesia, acute thoracotomy, and pericardiotomy, heart rate and blood pressure increased, mean values $156 \pm 10$ beats $/ \mathrm{min}(P<0.01)$ and $117 \pm 4 \mathrm{~mm} \mathrm{Hg}(P<0.01)$, and blood flow increased in both the epicardium, 1.08 \pm 0.10 , and endocardium, $1.11 \pm 0.08 \mathrm{~cm}^{3} / \mathrm{min} / \mathrm{g}(P<0.01)$. Blood flow values in comparable epicardial and endocardial regions of rings 2 and 3 were not different statistically, with the exception of the septal endocardium where blood flow in ring 2 was significantly higher than in ring 3. Values from comparable regions were averaged in each dog, except for the septal endocardium, and then subjected to multivariate analysis to test for possible differences between regional blood flow (column C, Table I). No significant differences were found in regional blood flow in the epicardium but differences were present in the endocardium, i.e., posterior, posterior papillary, lateral, and anterior papillary endocardial flows were significantly greater than anterior endocardial flow (column C, Table I). Analysis of septal endocardial flow indicated that blood flows in rings 2 and 3 were greater than and equal to anterior endocardial blood flow, respectively. When compared to measurements before anesthesia, mean endo/epi ratios decreased in each region with the exception of posterior papillary region; the ratios were statistically greater than 1.0 in both papillary muscle regions (column C, Table II). Thus, anesthesia, thoracotomy, and pericardiotomy were associated with redistribution of transmural blood flow since there was less increase in endocardial than epicardial flow.

Adenosine was infused intravenously after anesthesia and acute surgery in nine dogs. Mean heart rate was $176 \pm 10$ beats/min and arterial pressure was $96 \pm 3 \mathrm{~mm}$ $\mathrm{Hg}$. Both values were significantly higher $(P<0.05)$ than corresponding values during adenosine infusion before acute anesthesia and surgery. Adenosine increased blood flow markedly in both the epicardium and endocardium, mean values were $5.19 \pm 0.57$ and $3.52 \pm 0.30$ $\mathrm{cm}^{3} / \mathrm{min} / \mathrm{g}$, respectively (each $P<0.01$ ). Epicardial blood flow values during adenosine before and after anesthesia and surgery were not statistically different $(P>0.1)$, while endocardial blood flow was significantly less after anesthesia and surgery $(P<0.01)$. Blood flow values in comparable epicardial and endocardial regions, including the septum, of rings 2 and 3 were not different statistically and thus were averaged and then subjected to multivariate analysis to test for differences between regional flow (column $\mathrm{D}$, Table $\mathrm{I}$ ). Regional epicardial blood flow was not significantly different, while endocardial flows in the posterior and posterior papillary muscle were significantly less than anterior endocardial flow. Transmural blood flow gradients in each region were reversed, i.e., endo/epi ratios varying from 0.63 to
0.78 (column D, Table II). Thus, the combination of adenosine and acute anesthesia and surgery resulted in marked redistribution of transmural blood flow.

\section{DISCUSSION}

Radioactive microspheres have been reported to yield values for flow which are comparable to simultaneous measurements of the following: $(a)$ renal and placental blood flow using electromagnetic flowmeters $(8,14)$, (b) cardiac output using both Fick and indicator dilution methods (15), and (c) myocardial flow obtained by measuring coronary venous return during right heart bypass $(8,16)$. The myocardial distribution of microspheres was studied by Domenech et al. (16) and reported to be a function of microsphere size as well as blood flow. Subendocardial trapping exceeded subepicardial trapping 2.5 times using 51-61 $\mu \mathrm{m}$ microspheres and 1.3 times using $14-\mu \mathrm{m}$ microspheres (16). These investigators concluded that neither size microspheres accurately estimated transmural blood flow. It was postulated that the higher subendocardial values were due to a tendency for these particles to stream axially, resulting in underperfusion of the epicardium and overperfusion of the endocardium. Subsequent studies were carried out in anesthetized animals using $7-10-\mu \mathrm{m}$ microspheres $(8,9)$. The endocardial and epicardial distribution of these smaller microsphere was not significantly different $(8,9)$ and thus was comparable to the endocardial and epicardial distribution of diffusable indicators (4-7). These findings suggest that the smaller microspheres are distributed in a manner similar to red blood cells. In addition, it has been determined previously and confirmed in the present study that the $7-10-\mu \mathrm{m}$ microspheres do not escape the coronary circulation since less than $1.0 \%$ of the myocardial radioactivity was observed in the coronary venous drainage (8). Thus, it seems reasonable to conclude that the distribution of $7-10-\mu \mathrm{m}$ microspheres may provide an accurate estimate of the true distribution of myocardial blood flow.

Buckberg et al. (8) observed that the primary measurement error of the microsphere technique was due to random variation in the distribution of microspheres. The samples studied included: reference blood, right and left ventricle, entire heart, and kidney. These workers reported that the measurement error was usually under $20 \%$ if greater than 400 microspheres were present in the sample and that the measurement error rose precipitously if the sample contained less than 400 microspheres (8). From this data, it seems reasonable to conclude that measurements of blood flow in smaller myocardial samples may be made with the same degree of precision if an adequate number of microspheres are used. In the present study, the number of microspheres injected was calculated to provide more than 500 micro- 
spheres/g of myocardium and each sample contained at least $1 \mathrm{~g}$ of tissue.

In the present study, blood flow differences or variability between adjacent samples in both the epicardium and endocardium, i.e. regions which may be expected to have the least differences in flow, were small, i.e., $95 \%$ confidence limits of $\pm 20 \%$. Blood flow differences were slightly greater during maximum flow rates. This variation between relatively small regions of the myocardium was comparable to the variability reported in measuring the distribution of microspheres to the right and left ventricle and in measuring blood flow to the entire heart (8). Since the magnitude of blood flow differences was small and within the measurement variability that was found in determining flow to the entire left ventricle (8), the results indicate that blood flow between relatively small adjacent epicardial and adjacent endocardial samples is essentially homogeneous. These results do not rule out the possibility that blood flow to smaller areas is heterogeneous (17).

No significant differences were demonstrated between blood flows to the regional areas of either the epicardium or the endocardium during quiet resting conditions, except for the septal endocardium. Septal endocardial flow in ring 2 was greater than septal endocardial flow in ring 3 in both the awake state and after anesthesia; however, maximum blood flow rates in these areas before and after anesthesia were not different. We have no explanation for this finding.

Previous data concerning the transmural distribution of blood flow during control conditions have been conflicting. Investigations using radioisotope depot clearance techniques $(2,3)$ have concluded that the endocardium receives less blood than the epicardium during control periods. Other investigators, using either diffusable indicators (4-7) or 7-10- $\mu \mathrm{m}$ microspheres, have reported that endocardial and epicardial flow measurements were not significantly different. Since wall tension at the endocardial surface is greater than at the epicardial surface (18), it is likely that the requirement for oxygen in the endocardium exceeds that of the epicardium. Because the capacity of the myocardium to increase extraction of oxygen is limited while the capacity for further vasodilation in the endocardium is considerable, as demonstrated in the present study, it seems reasonable that the greater oxygen needs should be met by greater myocardial flow. In the present study, during resting conditions significant transmural flow gradients were present in most regions, i.e., the endocardium receiving $6-16 \%$ more flow than the epicardium. Failure to observe a significant transmural flow gradient with $7-10-\mu \mathrm{m}$ microspheres or with the diffusable indicators used in the previous reports may be related to anesthesia and/or the altered hemodynamic state as- sociated with acute surgical trauma. In the present study, pentobarbital anesthesia, thoracotomy, and the pericardiotomy resulted in increases in blood flow in both the epicardium and endocardium, but the increase was less in the endocardium, resulting in a decrease or obliteration of transmural gradients present during control conditions. The mean heart rate increased from 71 to 156 beats/min after anesthesia and surgery and this tachycardia may have been a factor which influenced redistribution of blood flow. In a recent report, Neill, Phelps, Oxendine, Mahler, and Sim (19), using 15- $\mathrm{m}$ microspheres to measure flow in tranquilized dogs, reported control endo/epi ratios of $1.26-1.39$ at heart rates of 71 beats/min which decreased to $1.07-1.18$ at heart rates of 193 beats/min. Buckberg et al. (8), using 7-10- $\mu \mathrm{m} \mathrm{mi-}$ crospheres in anesthetized dogs, control heart rate 167 beats/min, reported no significant change in the subendocardial/subepicardial ratio when the rate was increased to 250 beats $/ \mathrm{min}$.

Winbury (1) using polarographic techniques for measuring intramyocardial oxygen tension and diffusable isotopes to estimate blood flow, concluded that under normal conditions the endocardium is not only underperfused but that the endocardial arteriolar resistance is fixed, resulting in no reserve capacity to increase flow by further vasodilation. Kirk and Honig (2), using depot clearance techniques, reported that in open-chest dogs endocardial flow was $25 \%$ less than epicardial flow; they concluded that the endocardial vessels failed to compensate for the pressure gradient which inhibited flow during systole. Buckberg, Fixler, Archie, and Hoffman (9), using 7-10- $\mu \mathrm{m}$ microspheres, observed that during rapid ventricular pacing total coronary flow increased $14 \%$, range -7 to $+69 \%$, and subendocardial and subepicardial blood flow increased equally and thus challenged the view that the subendocardial vessels are maximally dilated during control conditions. In the present study, blood flow in both the epicardium and endocardium increased markedly during adenosine infusion, indicating a considerable reserve capacity for vasodilation in each layer.

It has been hypothesized that the endocardium receives little or no blood during systole as a result of the systolic compression of the intramyocardial vasculature and compensation for this deficit in systolic flow is effected by greater vasodilation of endocardial vessels during diastole. The net effect of this relationship is to maintain adequate endocardial flow but at a reduced capacity to further increase endocardial flow (10). Data obtained in the present study during maximum coronary vasodilation evoked by adenosine lend support to this hypothesis. Whereas adenosine infusion markedly increased flow to both the endocardium and epicardium, endocardial flow was increased to a lesser degree than 
epicardial flow. Alternatively, Buckberg and associates (9) have emphasized the effects of coronary perfusion pressure (aortic diastolic minus left ventricular diastolic pressure) and the duration of diastole as determinants of endocardial perfusion. Adenosine infusion significantly increased heart rate, mean 40 beats $/ \mathrm{min}$, and resulted in modest decreases in arterial pressure, mean $14 \mathrm{~mm} \mathrm{Hg}$. Thus, the decrease in pressure and decrease in the period of diastole resulting from the increase in heart rate may have contributed to the smaller increase in endocardial blood flow during adenosine-induced vasodilation.

Although anesthesia and surgery did not alter the ability of the epicardial blood flow to increase in response to adenosine infusion, the increase in endocardial blood flow produced by adenosine was $22-37 \%$ less than the increase in epicardial flow. The smaller increase in endocardial flow during adenosine infusion after anesthesia occurred despite a significantly higher arterial pressure than during adenosine infusion before anesthesia. Although adenosine produced comparable increases in heart rate before and after anesthesia, the heart rate was considerably higher after anesthesia and surgery. It is likely that shortening of the diastolic perfusion period during tachycardia may have a greater effect on transmural distribution of blood flow during very high flow than during normal flow rates. During submaximal flow rates, shortening of the diastolic perfusion period may be matched by decreases in vasomotor tone in the endocardium but during maximal flow vasomotor tone is minimal and shortening of the diastolic perfusion period results in reduction in flow to areas which are perfused predominantly during diastole. Although anesthesia and acute surgery further decreased the capacity to augment blood flow in the endocardium, a significant capacity remained even after these interventions.

\section{ACKNOWLEDGMENTS}

The authors wish to acknowledge the following individuals who rendered valuable assistance in carrying out this study: Drs. Philip A. McHale and C. Frank Starmer for assistance with the statistical analysis; Dr. Judith C. Rembert for assistance with the radioisotope measurements; Mr. Kirky Cooper, Mr. Eric Fields, Mr. James Ferrell, and $\mathrm{Mr}$. George Kelser for expert technical assistance; Mr. J. Michael Taylor and his staff of the Durham Veterans Administration Hospital Animal Care Facility; Mr. Donald G. Powell of the Durham Veterans Administration Hospital Medical Illustration Department; and Mrs. Rosa B. Ethridge for secretarial assistance.

This work was supported by Veterans Administration Project no 1500-01 and by National Institutes of Health grant HL-09711 from the U. S. Public Health Service.

\section{REFERENCES}

1. Winbury, M. M. 1971. Redistribution of left ventricular blood flow produced by nitroglycerin. An example of integration of the macro- and microcirculation. Circ. Res. 28: Suppl. I: 140.

2. Kirk, E. S., and C. R. Honig. 1964. Nonuniform distribution of blood flow and gradients of oxygen tension within the heart. Am. J. Physiol. 207: 661.

3. Brandi, G., W. M. Fam, and M. McGregor. 1968. Measurement of coronary flow in local areas of myocardium using xenon ${ }^{138}$.J. Appl. Physiol. 24: 446.

4. Cutarelli, R., and M. N. Levy. 1963. Intraventricular pressure and the distribution of coronary blood flow. Circ. Res. 12: 322.

5. Moir, T. W., and D. W. DeBra. 1967. Effect of left ventricular hypertension, ischemia, and vasoactive drugs on the myocardial distribution of coronary flow. Circ. Res. $21: 65$.

6. Griggs, D. M., Jr., and Y. Nakamura. 1968. Effect of coronary constriction on myocardial distribution of iodoantipyrine--131. Am. J. Physiol. 215: 1082.

7. Love, W. D., and G. E. Burch. 1957. Differences in the rate of $\mathrm{RB}^{\mathrm{se}}$ uptake by several regions of the myocardium of control dogs and dogs receiving L-norepinephrine or Pitressin. J. Clin. Invest. 36: 479.

8. Buckberg, G. D., J. C. Luck, B. Payne, J. I. E. Hoffman, J. P. Archie, and D. E. Fixler. 1971. Some sources of error in measuring regional blood flow with radioactive microspheres. J. Appl. Physiol. 31: 598.

9. Buckberg, G. D., D. E. Fixler, J. P. Archie, and J. I. E. Hoffman. 1972. Experimental subendocardial ischemia in dogs with normal coronary arteries. Circ. Res. $30: 67$.

10. Moir, T. W. 1972. Subendocardial distribution of coronary blood flow and the effect of antianginal drugs. Circ. Res. 30: 621 .

11. Wolf, M. M., and R. M. Berne. 1956. Coronary vasodilator properties of purine and pyrimidine derivatives. Circ. Res. $4: 343$.

12. Morrison, D. F. 1967. Multivariate Statistical Methods. McGraw-Hill Book Co., New York. 117.

13. Miller, R. G., Jr. 1966. Simultaneous Statistical Inference. McGraw-Hill Book Co., New York. 67.

14. Rudolph, A. M., and M. A. Heymann. 1967. Circulation of the fetus in utero. Methods for studying distribution of blood flow, cardiac output and organ blood flow. Circ. Res. 21: 163.

15. Hoffbrand, B. I., and R. P. Forsyth. 1969. Validity studies of the radioactive microsphere method for the study of the distribution of cardiac output, organ blood flow, and resistance in the conscious rhesus monkey. Cardiovasc. Res. $3: 426$.

16. Domenech, R. J., J. I. E. Hoffman, M. I. M. Noble, K. B. Saunders, J. R. Henson, and S. Subijanto. 1969. Total and regional coronary blood flow measured by radioactive microspheres in conscious and anesthetized dogs. Circ. Res. 25: 581.

17. Bassingthwaighte, J. B., W. A. Dobbs, Jr., and T. Yipintsoi. 1972. Heterogeneity of myocardial blood flow. In Myocardial Blood Flow in Man, Methods and Significance of Coronary Disease. A. Maseri, editor. Minerva Medica, Torino. 197.

18. Streeter, D. D., Jr., R. N. Vaishnav, D. J. Patel, H. M. Spotnitz, J. Ross, Jr., and E. H. Sonnenblick. 1970. Stress distribution in the canine left ventricle during diastole and systole. Biophys. J. 10: 345 .

19. Neill, W. A., N. C. Phelps, J. M. Oxendine, D. J. Mahler, and D. N. Sim. 1973. Effect of heart rate on coronary blood flow distribution in dogs. Am. J. Cardiol. $32: 306$. 